\title{
Velocity Free Platoon Formation Control for Unmanned Surface Vehicles with Output Constraints and Model Uncertainties
}

\author{
Duansong Wang ${ }^{1 \oplus}$, Mingyu Fu ${ }^{1, *}$, Shuzhi Sam $\mathrm{Ge}^{2}$ and Dongyu $\mathrm{Li}^{3}{ }^{3}$ \\ 1 Colledge of Automation, Harbin Engineering University, Harbin 150001, China; \\ wangduansong@hrbeu.edu.cn \\ 2 Department of Electrical and Computer Engineering, University of Singapore, Singapore 119077, Singapore; \\ samge@nus.edu.sg \\ 3 Department of Control Science and Engineering, Harbin Institute of Technology, Harbin 150006, China; \\ lidy@hit.edu.cn \\ * Correspondence: fumingyu@hrbeu.edu.cn
}

Received: 3 November 2019; Accepted: 3 February 2020; Published: 7 February 2020

\begin{abstract}
This paper studies the velocity free platoon formation control for unmanned surface vehicles (USVs) with the model uncertainties and output constraints. Firstly, a reconstruction module is designed to estimate the velocity of the leader, which will be completed in finite time and will reduce the communication burden. Along with this, the model-based control combined with the symmetric barrier Lyapunov functions (BLF) method is designed to guarantee the output constraints. Then, the model uncertainties of the USV are approximated by the neural networks (NNs) and the NN BLF control is developed. To achieve the desired formation pattern, the constraints, including collision avoidance and communication distance, are under consideration. Finally, we proved that our system is semiglobally uniformly ultimately bounded (SGUUB) and verified the effectiveness of this approach by simulations.
\end{abstract}

Keywords: barrier Lyapunov functions; neural networks; output constraints; platoon formation control; unmanned surface vehicles

\section{Introduction}

In recent years, USV formation control has attracted large amounts of attention in the control science and engineering areas for different purposes such as mine clearance, patrol, investigation, transportation of strategic materials, coordinated operations, target rounding and marine science applications [1-4]. The objectives of formation control are to achieve the desired distance and orientation among multiple agents. Meanwhile, to avoid collision and keep effective communication during the formation control, the relative distance among USV should be guaranteed to be within a reasonable interval. Therefore, it is necessary to deal with model uncertainties and constraints to design the control system. A motivating example is controlling a group of moving cars in the form of closely spaced vehicular platoons while ensuring safety [5]. Vehicular platoons could deal well with traffic flow capacity and reduce the consumption of fuel in the automated highway systems. Therefore, many researchers extend the platoon formation control problem to robots or autonomous vehicles [5-7].

Great progress has been made in related research [8-12]. A control scheme based on the backstepping method technique and the Lyapunov direct method for formation control of ships using the leader-follower strategy was proposed in [13]. Based on [13], a control strategy was developed in [14] based on the bioinspired method for the underactuated surface vehicles that solved the problem of computation exploration and realized the constraints on virtual velocity. However, 
the ship models they used were all simplified and the model uncertainties were not considered. The unmodeled dynamics and parameter uncertainties induced by hydrodynamic damping terms may lead to problems that make it difficult or impossible to build an accurate ship model and this affects the transient and steady-state performance which leads to collisions and gives rise to invalid connectivity in formation control. In [15], the NN-based DSC technique was employed to solve uncertain local dynamics of leader-follower formation control. The adaptive NNs were used in [16] to deal with model uncertainties. The extended dissipativity criterion for a discrete-time $\mathrm{NN}$ with time-varying delay was established in [17]. An NN formation control for multiple USV cooperated with adaptive filtering methods were employed to extract the low-frequency content of the model uncertainties and ocean disturbances in [18]. The input constraint, model uncertainties, and external disturbances were considered for spacecraft formation control in [19]. Though the uncertain parameters and unmodeled dynamics or input constraints were considered in the above research, they have not taken the output constraints into consideration. The output and error constraints of the formation are very critical factors affecting formation stability, for the reason that if the distance between the two vehicles is too large and exceeds the effective range of communication in the process of formation pattern establishing, the connectivity between each vehicle will be invalid which will affect the formation pattern. On the other hand, if the distance between them is too short, it will cause intervehicle collisions and damage the system. Therefore, it is necessary to consider the output constraint of formation control. To deal with the problem of constraint, some methods have also been proposed to achieve the desired performance [6,20-22]. For example, the exponentially decaying functions of time and formation error transformation were used in [6]. An output feedback control was proposed in [23] for a certain class of systems that guaranteed the convergence of generic bounded solutions to the desired set-point. Under these methods, the formation errors are always within the predefined regions. To deal with the issue of connectivity preserving and collision avoidance problems between each other, a linear transformed error surface was introduced in [24]. To prevent output constraint violation, a symmetric BLF was employed in [25] and trajectory tracking with output constraints was realized. A two layered optimal approach towards cooperative motion planning of a USV in a constrained maritime environment was proposed in [26]. Reference [27] integrated the modules of efficient optimal path planning, robust path following guidance and the cooperative swarm aggregation approach towards development of a new hybrid framework for cooperative navigation of swarm of USVs to enable optimal and autonomous operation in a maritime environment.

Moreover, the information exchange between vehicles is a challenging problem. Most of the current research assumes that the full states of the leader can be accessed by the follower. The adaptive finite-time decentralized control problem was addressed in [28] with the time-varying output-constraint. Under the consideration of input quantization and dynamic uncertainties, a decentralized adaptive NN PI tracking control was developed for the interconnected nonlinear system in [29]. A new estimate-based dynamic event-triggered output feedback control for networked control systems subjected to nonlinear uncertainties was proposed in [30]. The BLF was used to solve the problem of state constraints for USV and the input saturation was considered in [31]. All the references listed need the full-state information of the leader or the desired trajectory. Actually, due to the reason of economy or efficiency for the formation control problem, only the position and heading can be obtained by the follower and the velocity information is unavailable. This implies that a practical control method which is less demanding for communications should be developed. A fuzzy state observer was introduced to estimate the unmeasurable state variables in [32]. An event-triggered $H \infty$ tracking control was first established for nonlinear NCSs in [33], which leads to a considerable reduction of sending data from the controller to the ROV. Unlike the existing studies in the previous literature, where the full states need to be known, this paper addresses the problem of the unavailability of the leader's velocity to the follower, which is more practical in modern applications.

The value of BLF will increase to infinity when its arguments are close to some specified value. So, keeping the BLF bounded could ensure that the constraints are never violated in the closed-loop 
system. Motivated by the above research and the property of BLF, a model-based formation control under the consideration of collision avoidance and effective communication distance constraints is proposed for USV, different from [6,24]. In this paper, the errors combined with a BLF are directly used to design the formation control instead of complex error conversion through the guaranteed performance function, which reduces the amount of calculation and simplifies the design of the control. Compared to existing work in this field, the main contributions of this paper are threefold.

- A BLF is used to tackle the problem of multiple USV constraints ensuring that the satisfactory formation performance with collision avoidance distance and connectivity constraints are never violated during the whole operation.

- An adaptive NN control combined with a symmetric BLF is developed for autonomous USV to deal with the unmodeled dynamics and parameter uncertainties caused by hydrodynamic damping terms.

- Unlike the existing studies in the previous literature, where the full states need to be known, this paper addresses the problem of the unavailability of the leader's velocity to the follower using a reconstruction module, which is more practical in modern applications. One of the contributions thus lies in that this method can accurately reconstruct the target velocity in finite time.

This paper is organized as follows. Section 2 introduces the preliminaries and problem description. Section 3 is devoted to model-based BLF control and NN adaptive control for multiple USV. Simulation examples verified the effectiveness of the proposed method in Section 4. Section 5 draws the conclusions of the work.

\section{Preliminaries and Problem Description}

\subsection{Preliminaries}

Lemma 1 ([25]). For any constant $x \in \mathbb{R}^{n}$, if $|x|<k, k$ is a constant, then the following inequality holds

$$
\ln \frac{k^{2}}{k^{2}-x^{2}} \leq \frac{x^{2}}{k^{2}-x^{2}}
$$

Lemma 2 ([34,35]). For an unknown continuous nonlinear function $f(x): \mathbb{R}^{m} \rightarrow \mathbb{R}$, the radial basis function $N N$ can be used to approximate it over a compact set $\Omega \subseteq \mathbb{R}^{m}$ as follows:

$$
f(x)=W^{* T} H(x)+\varepsilon(x),
$$

where $x \in \mathbb{R}^{n}$ is the input vector, $H(x)=\left[h_{1}(x), \ldots h_{m}(x)\right]^{T}$ is the radial basis function vector and $h_{j}=$ $\exp \left(-\frac{\left\|\mathbf{x}-c_{j}\right\|^{2}}{2 b_{j}^{2}}\right) . c=\left[c_{i j}\right]_{n \times m}$ is the center and $b_{j}$ is the width of the neural cell of the hidden layer. $\varepsilon(x)$ denotes the approximate error satisfying $\varepsilon(x) \leq \bar{\varepsilon}, \bar{\varepsilon}$ is an arbitrary small positive constant. The structure of the $N N$ is presented in Figure 1. The RBF optimal weight vector is $W^{*}=\left[w_{1}^{*}, \cdots, w_{m}^{*}\right]^{T}$. The weight vector of $W^{*}$ is calculated by

$$
W^{*}=\arg \min _{\hat{W}}\left\{\sup _{x \in \Omega}\left|f(x)-\hat{W}^{T} H(x)\right|\right\},
$$

where $\hat{W}$ is the estimate value of $W^{*}$, which is produced by an adaptive update law.

\subsection{USV Model}

Consider a class of networked multiagent systems consisting of $n+1$ USV (labeled as 0 to $n$ ) moving in a special formation pattern as shown in Figure 2. The motion of the $i$-th USV can be described with kinematics

$$
\dot{\eta}_{i}=J_{i}\left(\eta_{i}\right) v_{i}
$$


and dynamics

$$
M_{i} \dot{v}_{i}=-C_{i}\left(v_{i}\right) v_{i}-D_{i}\left(v_{i}\right) v_{i}+w_{i}+\tau_{i}+\Delta_{i}
$$

where $\eta_{i}=\left[x_{i}, y_{i}, \psi_{i}\right]^{T}$ represents the position and yaw angle of the USV in the earth-fixed frame. $J_{i}\left(\eta_{i}\right)$ is a nonsingular transformation matrix defined as $J_{i}\left(\eta_{i}\right)=\left[\begin{array}{ccc}\cos \left(\psi_{i}\right) & -\sin \left(\psi_{i}\right) & 0 \\ \sin \left(\psi_{i}\right) & \cos \left(\psi_{i}\right) & 0 \\ 0 & 0 & 1\end{array}\right] \cdot v_{i}=$ $\left[u_{i}, v_{i}, r_{i}\right]^{T}$ denotes the surge, sway and yaw velocities with respect to body fixed frame. $M_{i} \in \mathbb{R}^{3 \times 3}$ is a symmetric positive definite inertia matrix, $C_{i}\left(v_{i}\right)$ is the matrix of Coriolis and centripetal terms, $D_{i}\left(v_{i}\right)$ is the damping matrix, $w_{i}$ is the external disturbances induced by wind, wave, and ocean currents, etc., and $\Delta_{i}$ represents the unmodeled dynamics. The control inputs of the $i$-th USV is denoted by $\tau_{i}$.

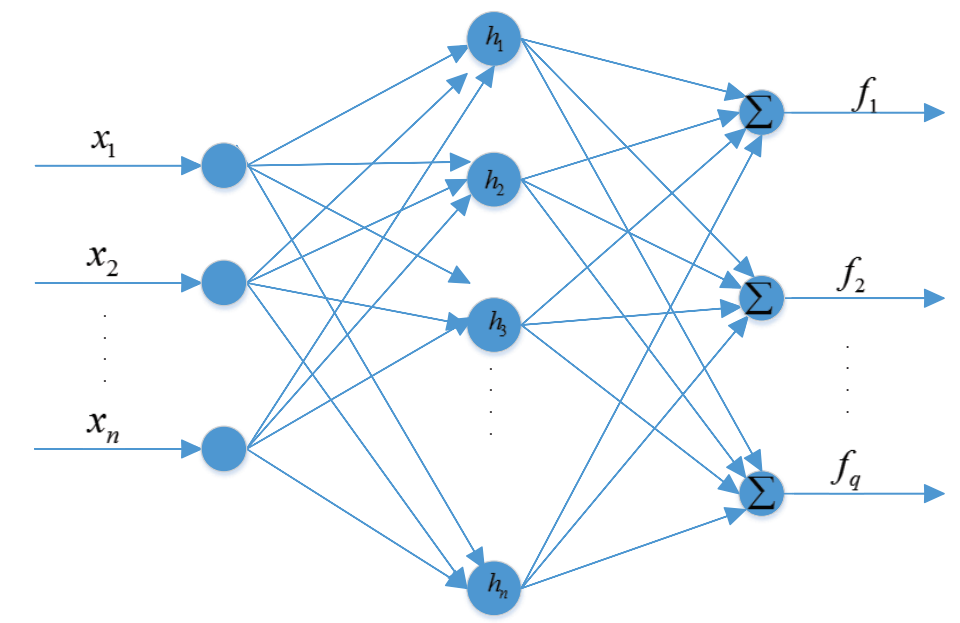

Figure 1. The structure of a neural networks (NN).

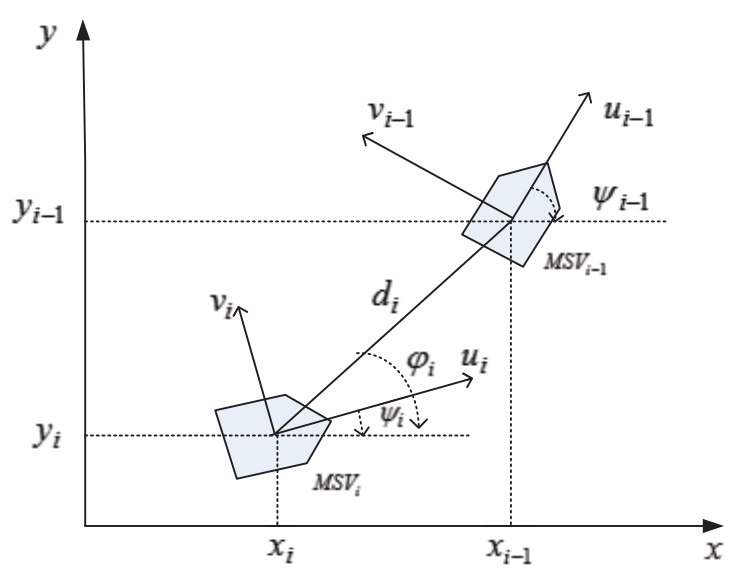

Figure 2. Unmanned surface vehicle (USV) formation configuration.

The matrices $M_{i}, C_{i}\left(v_{i}\right)$ and $D_{i}\left(v_{i}\right)$ are given below:

$$
\begin{gathered}
M_{i}=\left[\begin{array}{ccc}
m_{11 i} & 0 & 0 \\
0 & m_{22 i} & m_{23 i} \\
0 & m_{32 i} & m_{33 i}
\end{array}\right], D_{i}\left(v_{i}\right)=\left[\begin{array}{ccc}
d_{11 i} & 0 & 0 \\
0 & d_{22 i} & d_{23 i} \\
0 & d_{32 i} & d_{33 i}
\end{array}\right], \\
C_{i}\left(v_{i}\right)=\left[\begin{array}{ccc}
0 & 0 & -m_{22 i} v_{i}-m_{23 i} r_{i} \\
0 & 0 & m_{11 i} u_{i} \\
m_{22 i} v_{i}+m_{23 i} r_{i} & -m_{11 i} u_{i} & 0
\end{array}\right],
\end{gathered}
$$


where

$m_{11 i}=m_{i}-X_{u i}, m_{22 i}=m_{i}-Y_{\dot{v} i}, m_{23 i}=m_{32 i}=m_{i} x_{g i}-Y_{\dot{r} i}, m_{33 i}=I_{z i}-N_{\dot{r} i}$,

$d_{11 i}\left(u_{i}\right)=-\left(X_{u i}+X_{|u i| u_{i}}\left|u_{i}\right|+X_{\text {uiuiui }} u_{i}^{2}\right), d_{22 i}\left(v_{i}, r_{i}\right)=-\left(Y_{v i}+Y_{|v i| v_{i}}\left|v_{i}\right|+Y_{|r i| v_{i}}\left|r_{i}\right|\right)$,

$d_{23 i}\left(v_{i}, r_{i}\right)=-\left(Y_{r i}+Y_{|v i| r_{i}}\left|v_{i}\right|+Y_{|r i| r_{i}}\left|r_{i}\right|\right), d_{32 i}\left(v_{i}, r_{i}\right)=-\left(N_{v i}+N_{|v i| v_{i}}\left|v_{i}\right|+N_{|r i| v_{i}}\left|r_{i}\right|\right)$,

$d_{33 i}\left(v_{i}, r_{i}\right)=-\left(N_{r i}+N_{|v i| r_{i}}\left|v_{i}\right|+N_{|r i| r_{i}}\left|r_{i}\right|\right)$,

the mass of the ship $i$ is denoted by $m_{i}$ and the $i$-th ship's inertia matrix in the body-fixed frame is denoted by $I_{z i}$.

\subsection{Platoon Formation Control with Output Constraints}

We can see a graphic decsription about the platoon formation control performing in a real-time strait in Figure 3. When USVs moves in a formation from a wide passage to a constrained channel, they have to change the pattern while keeping connectivity and avoiding collision. With our proposed method, the desired performance can be achieved. In this paper, we consider a control strategy to achieve the satisfactory desired distance and angle among USVs with the intervehicle communications. Under the designed control for each vehicle of a string of $n+1$ USVs, the distance and angle errors can be constrained in a certain range. The line-of-sight (LOS) strategy [36] is used to define the distance and angle between the two USVs,

$$
\begin{aligned}
& d_{i}=\sqrt{\left(x_{i-1}-x_{i}\right)^{2}+\left(y_{i-1}-y_{i}\right)^{2}}, \\
& \varphi_{i}=\arctan 2\left(y_{i-1}-y_{i}, x_{i-1}-x_{i}\right) .
\end{aligned}
$$

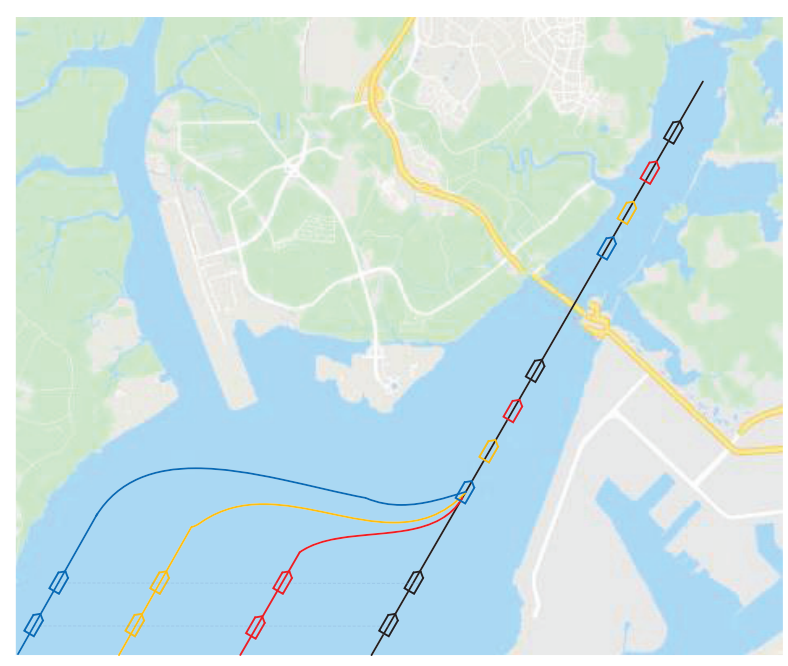

Figure 3. Platoon formation control graphic description in a real-time strait.

In order to avoid collision among vehicles, the desired distance during the whole moving process must satisfy the following equations:

$$
d_{i, \min \mathrm{col}}<d_{i} \leq d_{i, \max c o m}
$$

where $0<d_{i, \min c o l}<d_{i, \max c o m}$. The minimum safety distance is denoted by $d_{i, \min c o l}$ and the maximum effective communication distance is denoted by $d_{i, \max c o m}$. Next, the formation errors of the USVs are designed [21]:

$$
\begin{aligned}
& e_{d i}=d_{i}-d_{i, d e s}, \\
& e_{\psi i}=\psi_{i-1}-\psi_{i},
\end{aligned}
$$

where $d_{i, \text { des }}$ is a desired distance between the two USVs, and $d_{i, d e s}$ satisfies the inequality $0<d_{i, \text { min col }}<$ $d_{i, d e s}<d_{i, \operatorname{maxcom}}$. For convenience, we define $\mathrm{e}_{d i}(t)=d_{i, c o l \text { min }}-d_{i, d e s}$ as the minimum error distance 
and $\bar{e}_{d i}(t)=d_{i, \text { con } \max }-d_{i, d e s}$ as the maximum error distance. To guarantee the output constraints, we need to ensure that all signals are bounded and that the errors defined in Equation (8) are not violated, i.e.,

$$
\begin{aligned}
& \mathrm{e}_{d i}<e_{d i}<\bar{e}_{d i}, \\
& \mathrm{e}_{\psi i}<e_{\psi i}<\bar{e}_{\psi i} .
\end{aligned}
$$

\section{Control Design with a Symmetric BLF}

\subsection{Leader'S Velocity Estimation}

Note that the velocity of the leader may be not always accessible in practical applications, in which case a fast convergent observer is designed to estimate the unavailable states. According to Equation (2), let the unknown leader's velocity $\dot{\eta}_{l}$ pass through a first order filter:

$$
\dot{\phi}=-k \phi+k \dot{\eta}_{l}
$$

where $k \in \mathbb{R}$ is a positive scalar. Suppose that $\dot{\eta}_{l}$ is a differentiable function with time-derivative $\chi$, the dynamics in Equaiton (9) can be rewritten in the form of a linear system as follows:

$$
\begin{aligned}
\dot{x}_{1} & =-k x_{1}+k x_{2}, \\
\dot{x}_{2} & =\chi, \\
y & =x_{1},
\end{aligned}
$$

where $x_{1}$ and $x_{2}$ are equal to $\phi$ and $\dot{\eta}_{l}$, respectively, $\chi$ is the unknown input while $\mathrm{y}$ is the measurable output of the linear system. The problem of estimating $\dot{\eta}_{l}$ can be formulated as observing the states of the linear system (10). The following observer can therefore be designed to exactly reconstruct the linear system (10) with finite-time:

$$
\begin{aligned}
& \dot{\hat{x}}_{1}=-k \hat{x}_{1}+k \hat{x}_{2}-k 1 \operatorname{sign}\left(\tilde{x}_{1}\right)-k 2 \tilde{x}_{1}, \\
& \dot{\hat{x}}_{2}=-k 3 \tilde{x}_{1}-k 4\lfloor\vartheta\rfloor^{\frac{p}{q}}-k 5 \operatorname{sign}(\vartheta),
\end{aligned}
$$

where $\tilde{x}_{1}=\hat{x}_{1}-x_{1}$ and $\tilde{x}_{2}=\hat{x}_{2}-x_{2}$ are the estimation errors and are denoted by $\tilde{x}=\left[\tilde{x}_{1}^{T}, \tilde{x}_{2}^{T}\right]^{T}$, $k_{i} \in \mathbb{R}, i=1, \ldots, 5$ are positive observer gains to be defined later. The variable $\vartheta$ is defined as $\vartheta=k_{1} \operatorname{sign}\left(\tilde{x}_{1}\right), p$ and $q$ are positive odd integers such that $p<q$.

Theorem 1. The observer designed above ensures that the estimate value can converge to its real value in a finite time, provided that the observer gains are selected as: $k_{1}>\max \left\{\underline{k}_{1}, \bar{k}_{1}\right\}$ and $k_{5} k-\gamma>0$, where $\underline{k}_{1}=$ $\left(k \frac{k_{4} \sqrt{3}+\sqrt{3} k_{5}+\gamma}{\lambda_{\min }(P)}+\delta_{0}\right)^{\frac{q}{q+p}}$ and $\bar{k}_{1}=\left(\frac{k\left(\sqrt{3} k_{5}+\gamma\right)+\delta_{0} \lambda_{\min }(P)}{\sqrt{3} k_{5}+\gamma+\delta_{0} \lambda_{\min }(P)}\right)^{\frac{q}{p}}$ where $\delta_{0}>0$ is a positive scalar, and $P$ is a positive definite matrix given as $P=\left[\begin{array}{cc}\left(k+k_{2}\right) I_{3} & -k_{3} I_{3} \\ k_{3} I_{3} & 0_{3}\end{array}\right]$.

Proof. Detailed proof can be found in [37].

\subsection{Control Design}

In the case where matrices $M_{i}, C_{i}\left(v_{i}\right)$ and $D_{i}\left(v_{i}\right)$ are all known, let $z_{1 i}=\left[z_{11 i}, z_{12 i}\right]^{T}=\left[e_{d i}, e_{\psi i}\right]^{T}$, and $z_{2 i}=v_{i}-\alpha_{i}=\left[z_{21 i}, z_{22 i}, z_{23 i}\right]^{T}$, where $\alpha_{i}=\left[\alpha_{1 i}, \alpha_{2 i}, \alpha_{3 i}\right]^{T}$ is a stabilizing function which will be outlined later. Consider the symmetric BLF candidate as

$$
V_{1 i}=\frac{1}{2} \log \frac{k_{a i}^{2}}{k_{a i}^{2}-e_{d i}^{2}}+\frac{1}{2} \log \frac{k_{b i}^{2}}{k_{b i}^{2}-e_{\psi i}^{2}},
$$


where $k_{a i}$ and $k_{b i}$ are positive constants used to constrain $e_{d i}$ and $e_{\psi i}$, i.e., $\left|e_{d i}\right|<k_{a i},\left|e_{\psi i}\right|<k_{b i}$, respectively. Differentiating $V_{1 i}$ with respect to time we have

$$
\dot{V}_{1 i}=\frac{e_{d i} \dot{e}_{d i}}{k_{a i}^{2}-e_{d i}^{2}}+\frac{e_{\psi i} \dot{e}_{\psi i}}{k_{b i}^{2}-e_{\psi i}^{2}}
$$

According to Equation (8), differentiating $e_{d i}$ and $e_{\psi i}$ with respect to time, we can obtain

$$
\begin{aligned}
\dot{e}_{d i}= & -\left(z_{21 i}+\alpha_{1 i}\right) \cos \left(\psi_{i}-\varphi_{i}\right) \\
& +\left(z_{22 i}+\alpha_{2 i}\right) \sin \left(\psi_{i}-\varphi_{i}\right)+\dot{x}_{i-1} \cos \varphi_{i}+\dot{y}_{i-1} \sin \varphi_{i}, \\
\dot{e}_{\psi i}= & \dot{\psi}_{i-1}-\left(z_{23 i}+\alpha_{3 i}\right) .
\end{aligned}
$$

The virtual control $\alpha_{i}$ is designed as follows:

$$
\begin{aligned}
\alpha_{1 i} & =\cos \left(\psi_{i}-\varphi_{i}\right)\left[k_{d i} e_{d i}\left(k_{a i}^{2}-e_{d i}^{2}\right)+\dot{x}_{i-1} \cos \varphi_{i}+\dot{y}_{i-1} \sin \varphi_{i}\right], \\
\alpha_{2 i} & =-\sin \left(\psi_{i}-\varphi_{i}\right)\left[k_{d i} e_{d i}\left(k_{a i}^{2}-e_{d i}^{2}\right)+\dot{x}_{i-1} \cos \varphi_{i}+\dot{y}_{i-1} \sin \varphi_{i}\right], \\
\alpha_{3 i} & =k_{\psi i} e_{\psi i}\left(k_{b i}^{2}-e_{\psi i}^{2}\right)+\dot{\psi}_{i-1} .
\end{aligned}
$$

Substituting Equations (14) and (15) into Equaiton (13) yields

$$
\dot{V}_{1 i}=-k_{d i} e_{d i}^{2}-k_{\psi i} e_{\psi i}^{2}+\Theta_{1 i}
$$

where

$$
\begin{aligned}
\Theta_{1 i}= & \frac{e_{d i}\left(-z_{21 i} \cos \left(\psi_{i}-\varphi_{i}\right)+z_{22 i} \sin \left(\psi_{i}-\varphi_{i}\right)\right)}{k_{a i}^{2}-e_{d i}^{2}} \\
& +\frac{e_{\psi i}\left(-z_{23 i}\right)}{k_{b i}^{2}-e_{\psi i}^{2}} .
\end{aligned}
$$

The speed constraints of $v_{i}$ are not considered in this work, so another Lyapunov candidate function is chosen as follows:

$$
V_{2 i}=V_{1}+\frac{1}{2} z_{2 i}^{T} M_{i} z_{2 i}
$$

Then the time differentiation of Equation (17) can be written as

$$
\begin{aligned}
\dot{V}_{2 i}= & -k_{d i} e_{d i}^{2}-k_{\psi i} e_{\psi i}^{2}+z_{2 i}^{T}\left(\tau_{i}-C_{i}\left(v_{i}\right) v_{i}\right. \\
& \left.-D_{i}\left(v_{i}\right) v_{i}+w_{i}+\Delta_{i}-M_{i} \dot{\alpha}_{i}\right)+\Theta_{1 i} .
\end{aligned}
$$

The model-based control law can be designed as:

$$
\begin{aligned}
\tau_{i}= & -K_{2 i} z_{2 i}+C_{i}\left(v_{i}\right) v_{i}+D_{i}\left(v_{i}\right) v_{i}-w_{i} \\
& +M_{i} \dot{\alpha}_{i}-\Theta_{2 i}+\Delta_{i},
\end{aligned}
$$

where

$$
\begin{aligned}
k_{2 i} & \in \mathbb{R}^{3 \times 3}>0, \\
\Theta_{2 i} & =\left[\begin{array}{lll}
\frac{e_{d i}\left(-\cos \left(\psi_{i}-\varphi_{i}\right)\right)}{k_{a i}^{2}-e_{d i}^{2}} & \frac{e_{d i} \sin \left(\psi_{i}-\varphi_{i}\right)}{k_{a i}^{2}-e_{d i}^{2}} & \frac{-e_{\psi i}}{k_{b i}^{2}-e_{\psi i}^{2}}
\end{array}\right]^{T} .
\end{aligned}
$$

Substituting Equation (19) into Equation (18), Equation (18) can be rewritten as

$$
\dot{V}_{2 i}=-k_{d i} e_{d i}^{2}-k_{\psi i} e_{\psi i}^{2}-k_{2 i} z_{2 i}^{T} z_{2 i}
$$


Based on Lemma 1, we can obtain that the error $e_{d i}$ can be constrained in the range of $\left(-k_{a i}, k_{a i}\right)$ and $e_{\psi i}$ could be constrained in the range of $\left(-k_{b i}, k_{b i}\right)$. However, the hydrodynamic damping $D_{i}\left(v_{i}\right) v_{i}$ is a continuous nonlinear function related to the USV speed. In addition, the unmodeled dynamics of the USV and model uncertainties have not been considered above. To solve these problems, an RBF $\mathrm{NN}$ is used to estimate the unknown dynamics and hydrodynamic damping terms. We give

$$
F_{i}\left(Z_{i}\right)=-D_{i}\left(v_{i}\right) v_{i}+\Delta_{i}
$$

where $F_{i}\left(Z_{i}\right)=\left[f_{1 i}\left(Z_{i}\right), f_{2 i}\left(Z_{i}\right), f_{3 i}\left(Z_{i}\right)\right]^{T} \in \mathbb{R}^{3}, Z_{i}=v_{i}$ is the input of the $\mathrm{NN}$, and $\Delta_{i}$ represents the unmodeled dynamics.

$$
f_{l i}\left(Z_{i}\right)=W_{l i}^{* T} H_{l i}\left(Z_{i}\right)+\varepsilon_{l i}\left(Z_{i}\right), l=1,2,3,
$$

where $W_{l i}^{*}$ is the true constant weight value, $H_{l i}\left(Z_{i}\right)$ is the RBF, $\varepsilon_{l i}\left(Z_{i}\right) \leq \bar{\varepsilon}_{i}\left(\bar{\varepsilon}_{i}>0\right.$ is an unknown arbitrary small constant, ) is the approximate error. The adaptive neural network control law is proposed as follows:

$$
\begin{aligned}
\tau_{i}= & -z_{2 i}^{+T}\left(\frac{k_{d i} e_{d i}^{2}}{k_{a i}^{2}-e_{d i}^{2}}+\frac{k_{\psi i} e_{\psi i}^{2}}{k_{b i}^{2}-e_{\psi i}^{2}}\right)+C_{i}\left(v_{i}\right)+M_{i} \dot{\alpha}_{i} \\
& -w_{i}-K_{2 i} z_{2 i}-\hat{W}_{i}^{T} H_{i}\left(Z_{i}\right)-\Theta_{2 i}
\end{aligned}
$$

where $(\bullet)^{+}$is the Moore-Penrose pseudoinverse of $(\bullet), \hat{W}_{i}=\left[\hat{W}_{1}, \hat{W}_{2}, \hat{W}_{3}\right]^{T}$ are the weights of the RBFNN, $\hat{W}_{i}^{T} H_{i}\left(Z_{i}\right)$ is used to approximate $W_{l i}^{* T} H_{l i}\left(Z_{i}\right)$.

$$
W_{l i}^{* T} H_{l i}\left(Z_{i}\right)=\hat{W}_{l i}^{T} H_{l i}\left(Z_{i}\right)-\varepsilon\left(Z_{i}\right)=-D_{i}\left(v_{i}\right) v_{i}+\Delta_{i}-\varepsilon\left(Z_{i}\right) .
$$

The adaptive update law is designed as follows:

$$
\dot{\hat{W}}_{i}=\Gamma_{i}\left(H_{i}\left(Z_{i}\right) z_{2 i}-\sigma_{i}\left|z_{2 i}\right| \hat{W}_{i}\right), i=1,2,3,
$$

where $\Gamma_{i}=\Gamma_{i}^{T}>0$ is the adaptive gain matrices and $\sigma_{i}$ is a positive constant.

Consider the Lyapunov function candidate

$$
V_{3 i}=V_{2 i}+\frac{1}{2} \sum_{i=1}^{3} \tilde{W}_{i} \Gamma_{i}^{-1} \tilde{W}_{i}
$$

where $\tilde{W}_{i}=\hat{W}_{i}-W_{i}^{*}$. Then the derivation of $V_{3 i}$ along Equations (18) and (25) is

$$
\begin{aligned}
\dot{V}_{3 i}= & -k_{d i} e_{d i}^{2}-k_{\psi i} e_{\psi i}^{2}+z_{2 i}^{T} \Theta_{2}+z_{2 i}^{T}\left(\tau_{i}-C_{i}\left(v_{i}\right)\right. \\
& \left.-M_{i} \alpha_{i}-w_{i}+W_{i}^{* T} H_{i}\left(Z_{i}\right)-\varepsilon_{i}\left(Z_{i}\right)\right)+\sum_{i=1}^{3} \tilde{W}_{i}^{T} \Gamma_{i}^{-1} \hat{W}_{i} .
\end{aligned}
$$

Substituting Equations (23), (24) and (25) into Equation (27), we can obtain

$$
\begin{aligned}
\dot{V}_{3 i}= & -k_{d i} e_{d i}^{2}-k_{\psi i} e_{\psi i}^{2}-\frac{k_{d i} e_{d i}^{2}}{k_{a i}^{2}-e_{d i}^{2}}-\frac{k_{\psi i} e_{\psi i}^{2}}{k_{b i}^{2}-e_{\psi i}^{2}} \\
& -z_{2 i}^{T} K_{2 i} z_{2 i}-z_{2 i}^{T} \varepsilon_{i}\left(Z_{i}\right)+\sum_{i=1}^{3} \tilde{W}_{i}^{T} \Gamma_{i}^{-1} \dot{\hat{W}}_{i}
\end{aligned}
$$


Then according to the Young inequality and Lemma 1, we have

$$
\begin{aligned}
\dot{V}_{3} \leq & -k_{d i} \log \frac{k_{a i}^{2}}{k_{a i}^{2}-e_{d i}^{2}}-k_{\psi i} \log \frac{k_{b i}^{2}}{k_{b i}^{2}-e_{\psi i}^{2}} \\
& -z_{2 i}^{T}\left(K_{2 i}-I\right) z_{2 i}+\frac{1}{2}\left\|\varepsilon_{i}\left(Z_{i}\right)\right\|^{2} \\
& +\sum_{i=1}^{3} \frac{\sigma_{i}^{2}}{8}\left(\left\|W_{i}^{*}\right\|^{4}+\left\|\tilde{W}_{i}\right\|^{4}-2\left\|W_{i}^{*}\right\|^{2}\left\|\tilde{W}_{i}\right\|^{2}\right) .
\end{aligned}
$$

According to Lemma 2, we can understand that

$$
\begin{aligned}
\left\|\tilde{W}_{i}\right\|= & \left\|\hat{W}_{i}-W_{i}^{*}\right\| \leq\left\|\hat{W}_{i}\right\|+\left\|W_{i}^{*}\right\| \\
& \leq \frac{s_{i}}{\sigma_{i}}+\left\|W_{i}^{*}\right\|=\mathfrak{\omega} .
\end{aligned}
$$

So we can obtain

$$
\dot{V}_{3 i} \leq-\rho V_{3 i}+C
$$

where

$$
\begin{aligned}
\rho & =\min \left(\min \left(2 k_{d i}, 2 k_{\psi i}\right), \frac{2 \lambda_{\min }\left(K_{2 i}-I\right)}{\lambda_{\max }\left(M_{i}\right)}, \min \left(\frac{\sigma_{i}^{2}\left\|W_{i}^{*}\right\|^{2}}{2 \lambda_{\max }\left(\Gamma_{i}^{-1}\right)}\right)\right) \\
C & =\frac{1}{2}\left\|\bar{\varepsilon}_{i}\right\|^{2}+\sum_{i=1}^{3}\left(\frac{\sigma_{i}^{2}}{8}\left\|W_{i}^{*}\right\|^{4}+\frac{\sigma_{i}^{2}}{8} \omega^{4}\right),
\end{aligned}
$$

the minimum and maximum eigenvalues of matrix $\bullet$ are denoted by $\lambda_{\min }(\bullet)$ and $\lambda_{\max }(\bullet)$, respectively. From Equation (31) we can obtain

$$
V_{3 i} \leq V_{3 i}(0)+\frac{C}{\rho}
$$

According to Equation (32), for $e_{d i}$ we have

$$
\log \frac{k_{a i}^{2}}{k_{a i}^{2}-e_{d i}^{2}} \leq 2\left(V_{3 i}(0)+\frac{C}{\rho}\right)
$$

Then, the distance error

$$
\left\|e_{d i}\right\| \leq \sqrt{k_{a i}^{2}-k_{a i}^{2} e^{2\left(V_{3 i}(0)+\frac{c}{\rho}\right)}} .
$$

Similarly, for angle error $e_{\psi i}$, we have

$$
\log \frac{k_{b i}^{2}}{k_{b i}^{2}-e_{\psi i}^{2}} \leq 2\left(V_{3 i}(0)+\frac{C}{\rho}\right),
$$

Then,

$$
\left\|e_{\psi i}\right\| \leq \sqrt{k_{d i}^{2}-k_{d i}^{2} e^{\left(2\left(V_{3 i}(0)+\frac{c}{\rho}\right)\right)}}
$$

Therefore, we can conclude that the errors $e_{d i}$ and $e_{\psi i}$ are SGUUB.

\section{Simulation Examples}

In this section, a string of four USVs are used for the numerical simulations to demonstrate the effectiveness of the proposed method. The communication relationship of the four USVs is shown in Figure 4. 


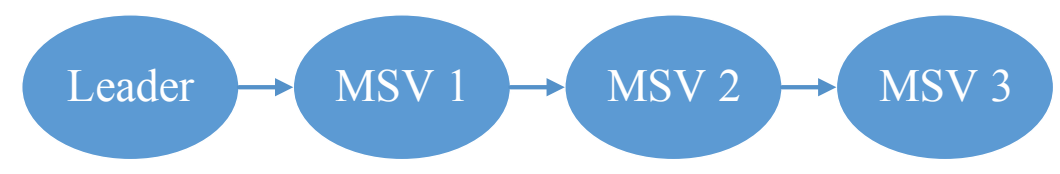

Figure 4. Communication graph among four USVs.

For the platoon formation control, every USV is a leader for its nearest successive USV except for the last one. So when an arbitrary USV is absent in practice, its follower should follow the virtual leader, which is at the forefront, and change the desired distance to one large enough. With this strategy, even if one of USVs is absent, the formation pattern and safety can be guaranteed.

The ship model parameters used for simulation in this work are those of Cybership-II, which is a 1:70 scale supply vessel replica built in a marine control laboratory in the Norwegian University of Science and Technology [38].

For the sake of convenience of comparison, the desired reference trajectory is chosen to be similar to [6],

$$
\eta_{0}=\left\{\begin{array}{l}
{[1.2 t, 0,0]^{T}, \text { if } t \leq t_{c},} \\
{\left[1.2 t_{c}+60 \sin t^{\prime}, 60\left(1-\cos t^{\prime}\right), t^{\prime}\right]^{T}, \text { if } t>t_{c},}
\end{array}\right.
$$

where $t_{c} \geq 0$ is a time constant and $t^{\prime}=0.02\left(t-t_{c}\right)$. The desired distance between the follower and the leader is considered to be $5 \mathrm{~m}$. The initial states of the USVs are given as $\eta_{0}=[0,0,0]^{T}$, $\eta_{1}=[0,5.9,0]^{T}, \eta_{2}=[0,11.8,0]^{T}, \eta_{3}=[0,17.7,0]^{T}$ and $v_{1}=v_{2}=v_{3}=0$. The time constant $t_{c}=200 \mathrm{~s}$. Figure 4 illustrates the validity of the position and velocity information among USVs. The maximum thrust force is $2 \mathrm{~N}$. In this paper, we consider the environment disturbances as $w_{i}=[0.1 \sin t, 0.1 \sin t, 0]^{T}$. The minimum collision distance is $4 \mathrm{~m}$ and the maximum connectivity distance is $6 \mathrm{~m}$. So the distance error should be satisfied by the following constraints:

$$
d_{i, \text { col } \min }<d_{i}<d_{i, \text { con } \max } \text {. }
$$

If the inequality is satisfied during the entire process of moving, it means that the collision distance and effective connectivity distance will not be violated, so the output constraint performance can be guaranteed. In order to verify the effectiveness of the proposed controller, two cases are evaluated for Matlab simulation studies.

Case I (model-based control): For the model-based control, the control parameters are chosen as follows: $k_{d 1}=12, k_{d 2}=k_{d 3}=1, k_{\psi 1}=1, k_{\psi 2}=k_{\psi 3}=0.5, k_{a 1}=k_{a 2}=k_{a 3}=1$ and $k_{b 1}=k_{b 2}=k_{b 3}=0.5$. The control gains are set to $K_{2 i}=\operatorname{diag}[6,6,4]$. The parameters $k_{a i}$ and $k_{b i}$ are the distance constraint and angle constraint selected based on the communication equipment or other plant requirements. The value of $k_{d i}$ and $k_{\psi i}$ affect the virtual control of $\alpha_{1 i}, \alpha_{2 i}$ and $\alpha_{3 i}$, if $k_{d i}$ and $k_{\psi i}$ are too large, the virtual control will become large which may lead to the control input violating the physical limit value and affecting the stability of the system. If $k_{d i}$ and $k_{\psi i}$ are too small, the control input will not drive the USV to the desired velocity.

Figure 5 shows that the unmeasurable surge, sway and yaw velocity of the leader USV can be measured by the designed finite time observer with zero estimate error. From Figure 6, it is obvious that the USVs can move in a desired formation pattern under the model-based method. We can observe that from Figure 7 the distance between the successive vehicles is limited to 4 (collision avoidance distance) to 6 (the maximum connection distance) during the whole moving process. So the distance error constraints $e_{d i}<k_{a i}$ and $e_{d i}>-k_{a i}, \forall t>0$ and relative angle error constraints $-k_{b i}<e_{\psi i}<k_{b i}$, $\forall t>0$ are realized. Figure 8 shows yaw angle tracking errors along with proposed boundary.

Case II (adaptive NN control): Secondly, to demonstrate the control integrated with NNs, we choose the formation pattern to be the same as in case I. The hydrodynamic damping matrix $D_{i}\left(v_{i}\right)$ is unknown. Simulation parameters are chosen as follows: $k_{d 1}=11$ and $k_{d 2}=k_{d 3}=3$. The NN inputs are chosen as $Z_{i 1}=u_{i}$ and $Z_{i 2}=Z_{i 3}=\left[v_{i}, r_{i}\right]^{T}$. 

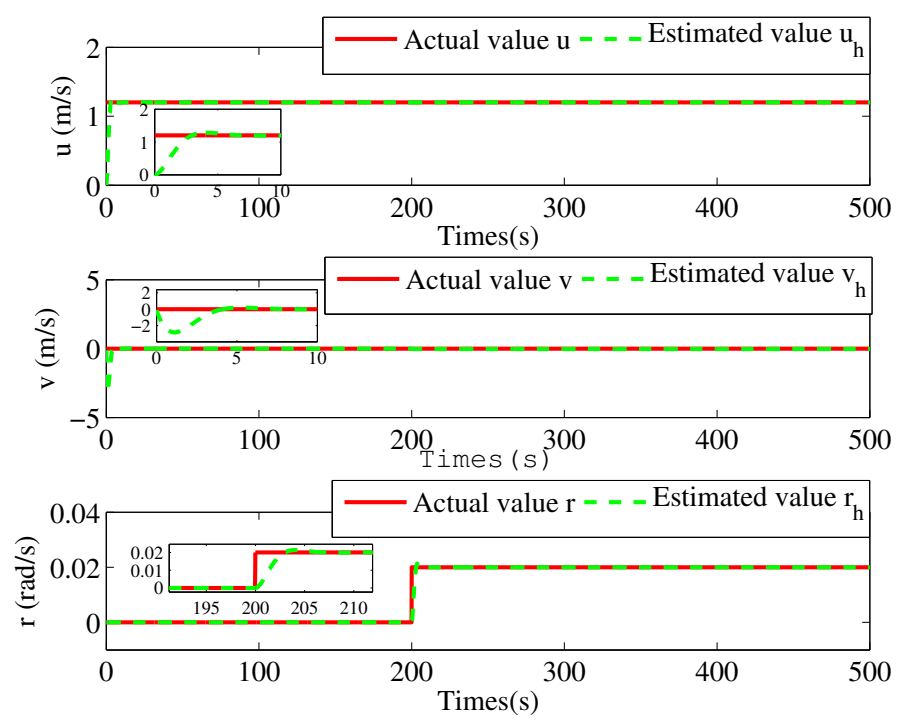

Figure 5. The estimate value of the finite time observer module.

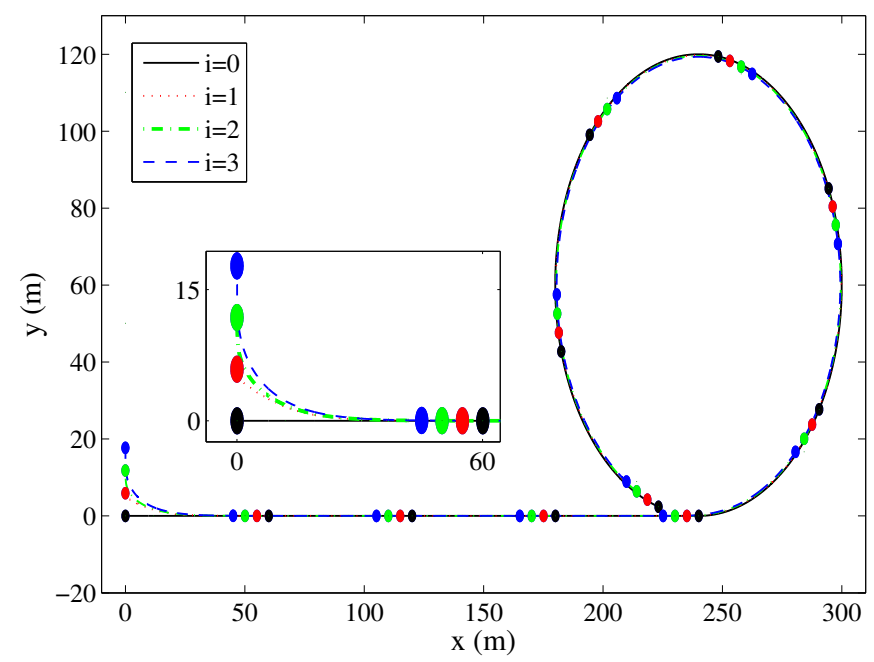

Figure 6. The north-east position of the four USVs under the model-based control.

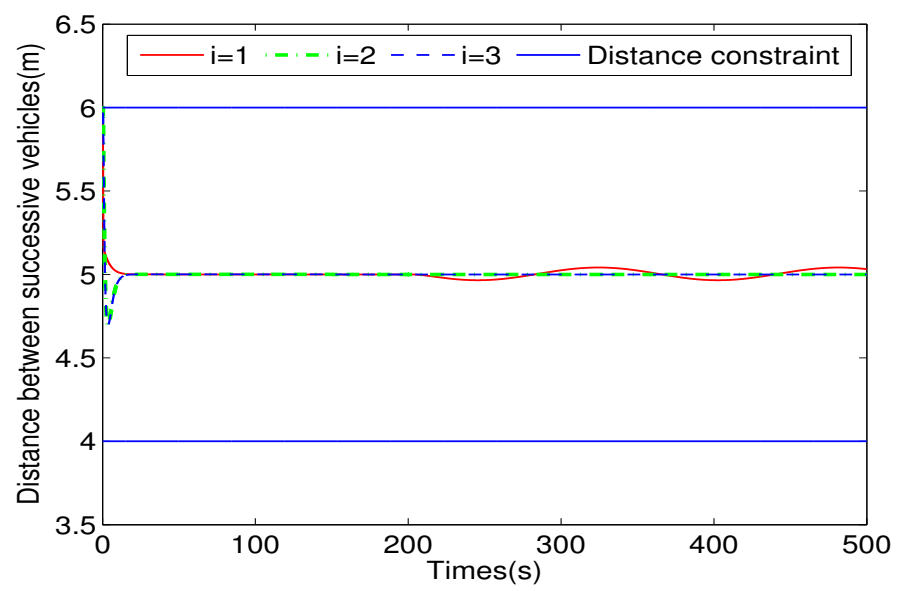

Figure 7. The line-of-sight (LOS) range $d_{i}$, the maximum connectivity distance and minimum collision distance under model-based control. 
The simulation results are shown in Figures 9-11. Figure 9 shows that the USVs are moving in the desired formation pattern. Figures 9-11 also show that though the hydrodynamic damping matrix $D_{i}$ is unknown to input $\tau_{i}$, through NN learning the LOS range $d_{i}$ converges to a small neighborhood of the desired distance and the error satisfies the predefined boundary restriction, which means that during the whole process the distance between the successive vehicles does not violate the collision and connectivity constraints. In addition, the idea of [39] is used to examine the robustness of the proposed method. To reduce the simulation time, only follower 1 is used to examine the robustness. The hydrodynamics damping term is randomly selected in the bound $\left[D^{*}-0.5 D^{*}, D^{*}+0.5 D^{*}\right]$, where $D^{*}$ is equal to the value in Equation (19), simulations are run for 100 sets of the parameters. The simulation results are shown in Figures 12 and 13. In addition, we also add the random Gaussian noise with amplitudes of $1 \mathrm{~cm}, 10 \mathrm{~cm}, 50 \mathrm{~cm}, 1 \mathrm{~m}, 1.2 \mathrm{~m}$ and $1.3 \mathrm{~m}$ to the trajectory of the leader, the control performance can be guaranteed below $1.2 \mathrm{~m}$, please see the simulation results in Figures 14 and 15 with the amplitudes of $1 \mathrm{~m}$ random Gaussian noise. It can be concluded that the proposed controller is so robust against parametric uncertainties and the USVs successfully achieve the desired formation pattern.

Remark 1. For the sake of convenience of comparison, the desired reference trajectory is chosen to be similar to that of [6]. Comparing Figures 6 and 9 with Figure 16, we can see that there is an overshoot in Figure 16 which is controlled by the method in [6]. In addition, in the Figures 6 and 9, the formation pattern can be achieved near $10 \mathrm{~m}$ on the $x$-axis, while it needs near $80 \mathrm{~m}$ in [6]. So the converge performance is better than the method proposed in [6].

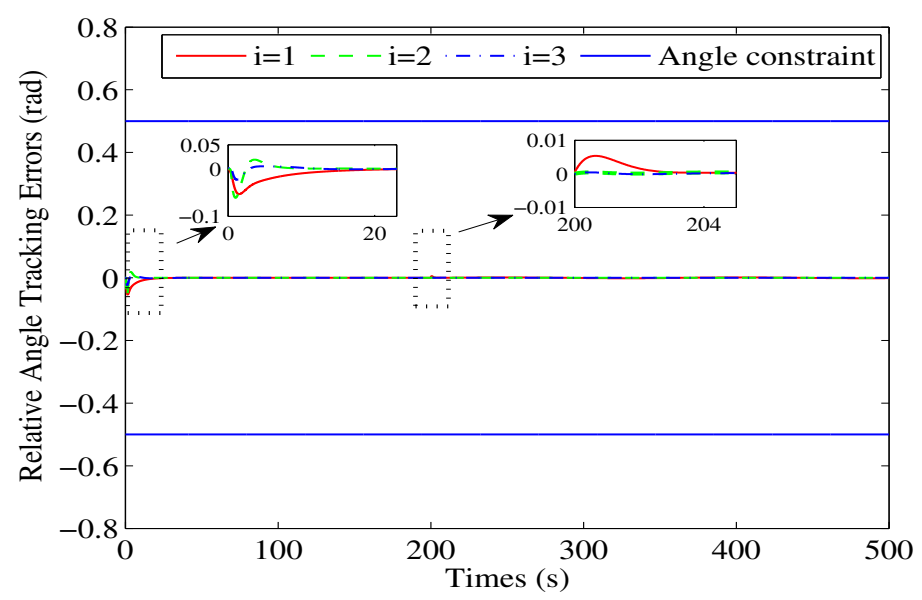

Figure 8. Yaw angle tracking errors along with proposed boundary.

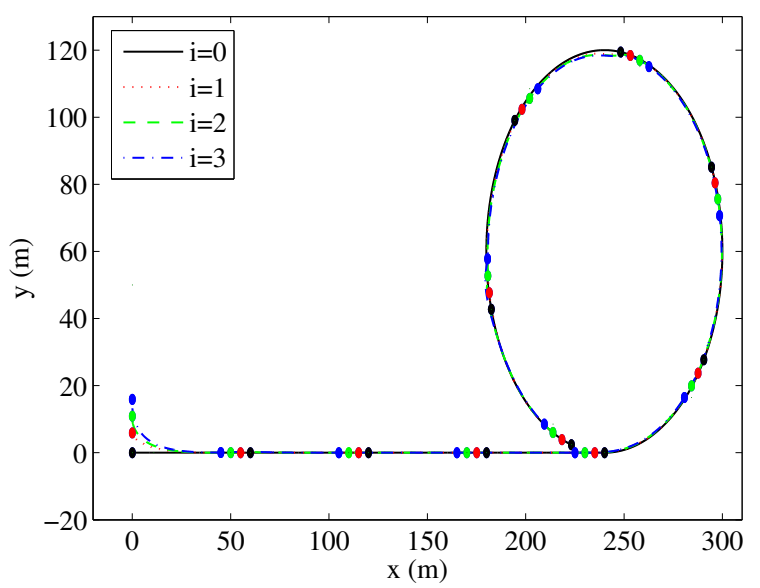

Figure 9. The north-east position of the four USVs under NN adaptive control. 


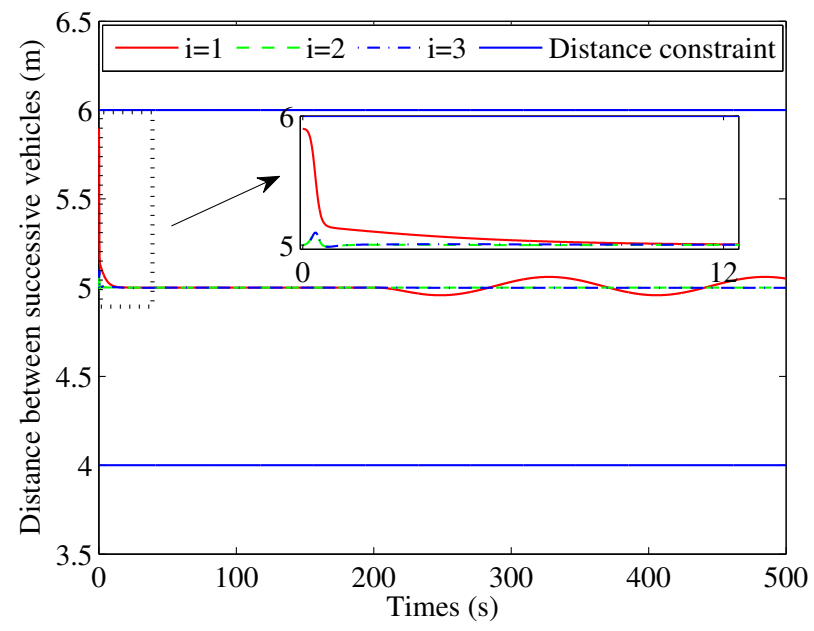

Figure 10. LOS range $d_{i}$ and the maximum connectivity distance and minimum collision distance under NN adaptive control.

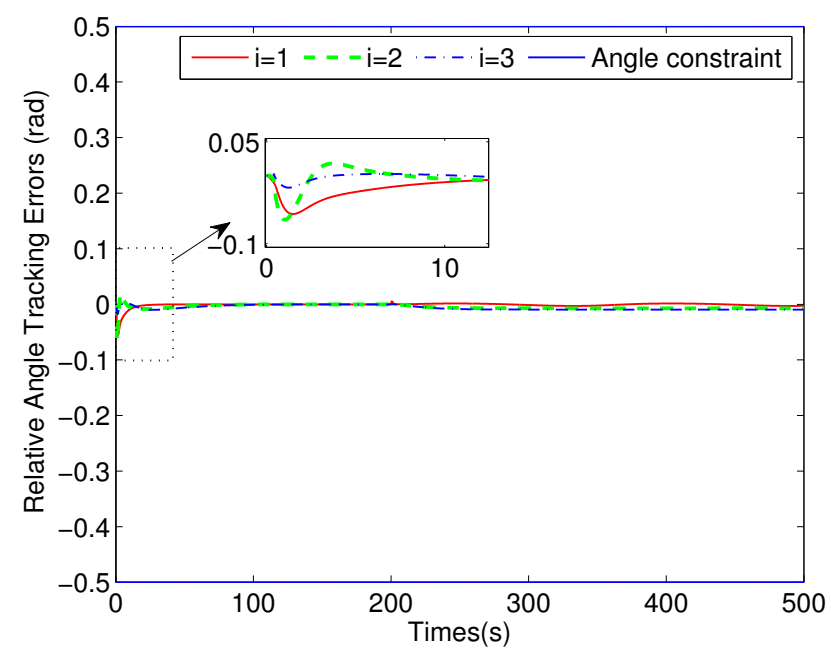

Figure 11. Yaw angle tracking errors along with proposed boundary.

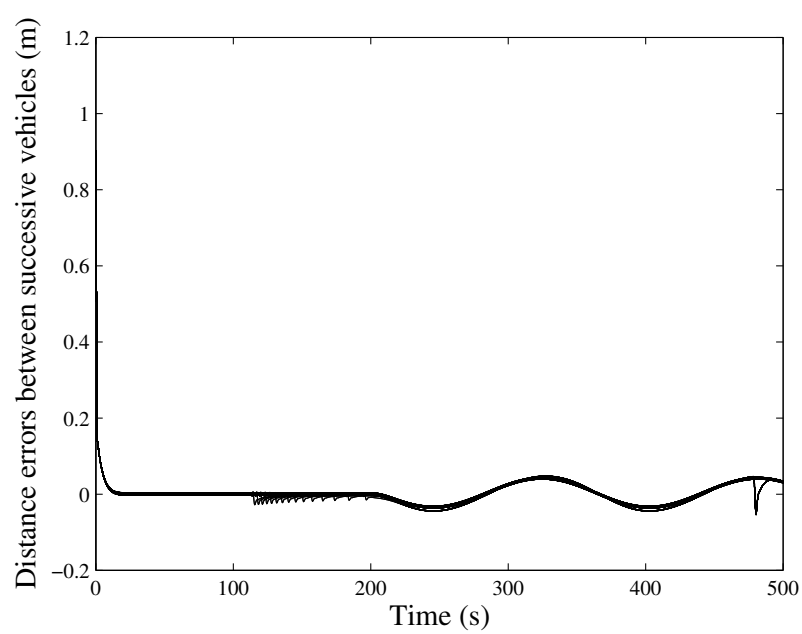

Figure 12. Graphs of the 100-set simulation distance errors. 


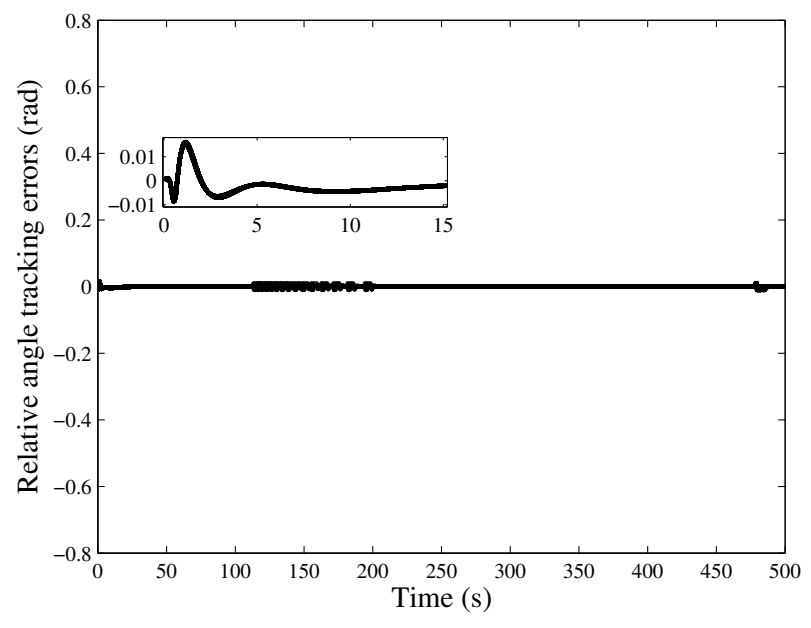

Figure 13. Graphs of the 100-set relative angle tracking errors.

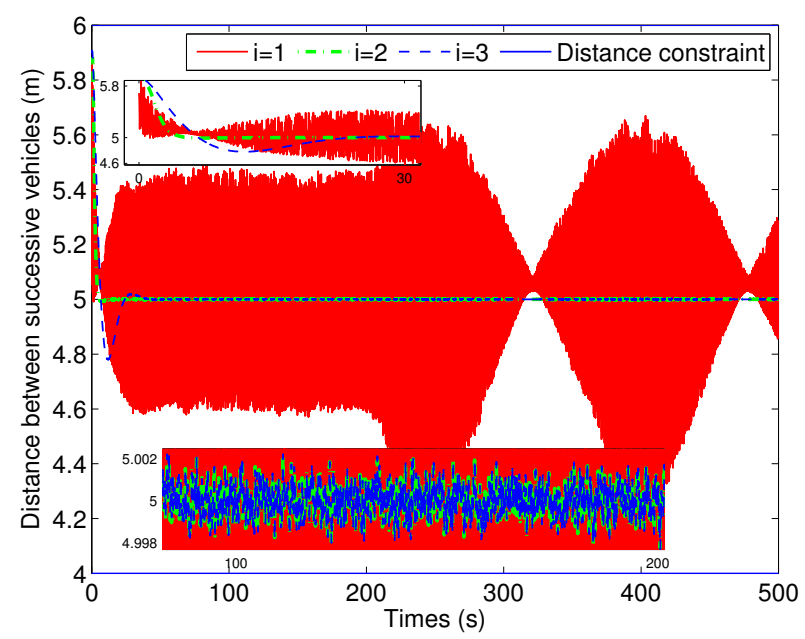

Figure 14. The LOS range $d_{i}$, the maximum connectivity distance and minimum collision distance under $1 \mathrm{~m}$ noise.

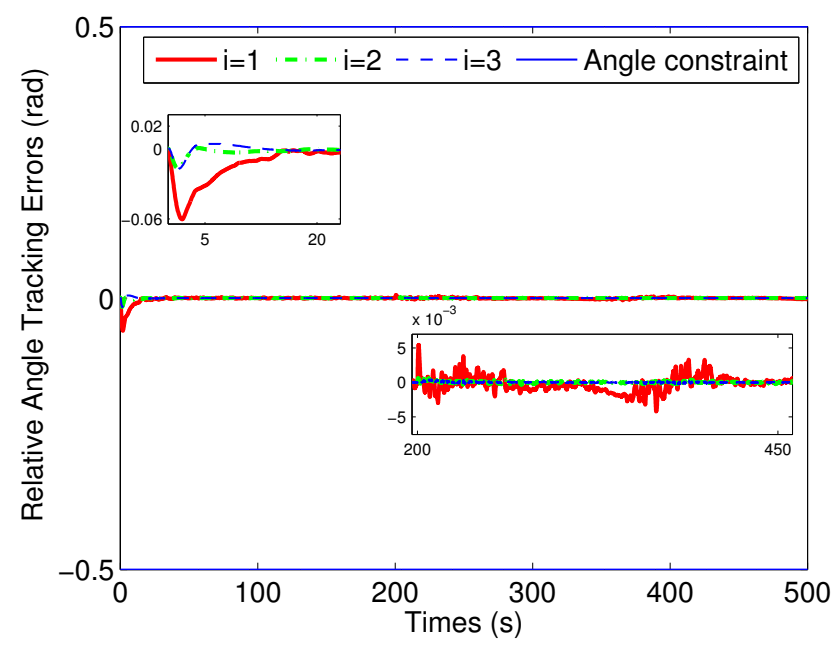

Figure 15. Yaw angle tracking errors under $1 \mathrm{~m}$ noise. 


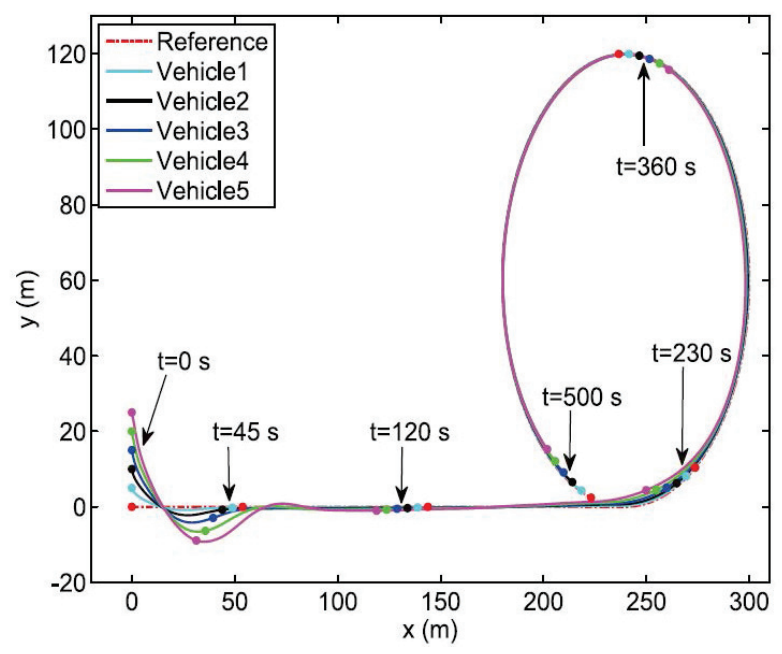

Figure 16. The north-east position of USVs under the method proposed in [6].

\section{Conclusions}

In this brief, a velocity-free formation control method for autonomous USVs with model uncertainties and output constraints is presented. Under the proposed model-based control, we have proven that the system is asymptotically stable and the LOS range and angle error constraints are never violated. Then under adaptive NN control, the system is proved to be SGUUB. Desired formation pattern is achieved without violation of the constraints including collision avoidance and connectivity distance. The simulation results generated confirm the performance of the proposed methods.

Author Contributions: Funding acquisition, M.F.; Methodology, D.W.; Software, D.W.; Supervision, M.F. and S.S.G.; Writing — original draft, Duansong Wang; Writing—review \& editing, D.L. All authors have read and agreed to the published version of the manuscript.

Funding: This research was funded in part by the National Nature Science Foundation of China grant number 51309062 and in part by the China Scholarship Council.

Conflicts of Interest: The authors declare no conflict of interest.

\section{Abbreviations}

The following abbreviations are used in this manuscript:

USV Unmanned surface vehicles

BLF Barrier Lyapunov function

RBF Radial basis function

NN Neural network

SGUUB semiglobally uniformly ultimately bounded

DSC Dynamic surface control

LOS Line-of-sight

\section{References}

1. Liu, Y.; Geng, Z. Finite-time optimal formation tracking control of vehicles in horizontal plane. Nonlinear Dyn. 2014, 76, 481-495. [CrossRef]

2. Nascimento, T.P.; Costa, L.F.S.; Conceição, A.G.S.; Moreira, A.P. Nonlinear Model Predictive Formation Control: An Iterative Weighted Tuning Approach. J. Intell. Robot. Syst. 2015, 80, 441-454. [CrossRef]

3. Wang, Q.; Wang, Y.; Zhang, H. The Formation Control of Multi-agent Systems on a Circle. IEEE/CAA J. Autom. Sin. 2018, 5, 148-154. [CrossRef]

4. Fan, Z.; Li, H. Two-layer model predictive formation control of unmanned surface vehicle. In Proceedings of the 2017 Chinese Automation Congress (CAC), Jinan, China, 20-22 October 2017; pp. 6002-6007. 
5. Horowitz, R.; Varaiya, P. Control design of an automated highway system. Proc. IEEE 2000, 88, 913-925. [CrossRef]

6. Dai, S.L.; He, S.; Lin, H.; Wang, C. Platoon Formation Control With Prescribed Performance Guarantees for USVs. IEEE Trans. Ind. Electron. 2018, 65, 4237-4246. [CrossRef]

7. Liu, Y.; Xu, B.; Ding, Y. Convergence Analysis of Cooperative Braking Control for Interconnected Vehicle Systems. IEEE Trans. Intell. Transp. Syst. 2016, 18, 1894-1906. [CrossRef]

8. Zhu, Q.; Ma, J.; Liu, Z.; Liu, K. Containment Control of Underactuated Ships with Environment Disturbances and Parameter Uncertainties. Math. Probl. Eng. 2017, 2017, 1-13. [CrossRef]

9. Maghenem, M.; Loria, A.; Panteley, E. A Cascades Approach to Formation-Tracking Stabilization of Force-Controlled Autonomous Vehicles. IEEE Trans. Autom. Control 2017, 63, 2662-2669.

10. Lu, Y.; Zhang, G.; Sun, Z.; Zhang, W. Robust adaptive formation control of underactuated autonomous surface vessels based on MLP and DOB. Nonlinear Dyn. 2018, 94, 503-519. [CrossRef]

11. He, W.; Chen, Y.; Yin, Z. Adaptive Neural Network Control of an Uncertain Robot With Full-State Constraints. IEEE Trans. Cybern. 2016, 46, 620-629. [CrossRef]

12. Li, D.; Zhang, W.; He, W.; Li, C.; Ge, S.S. Two-Layer Distributed Formation-Containment Control of Multiple Euler-Lagrange Systems by Output Feedback. IEEE Trans. Cybern. 2019, 49, 675-687. [CrossRef] [PubMed]

13. Ding, L.; Guo, G. Formation control for ship fleet based on backstepping. Control Decis. 2012, 27, $299-303$.

14. Fu, M.; Wang, D.; Wang, C. Formation Control for Water-Jet USV Based on Bio-Inspired Method. China Ocean Eng. 2018, 32, 117-122. [CrossRef]

15. Peng, Z.; Wang, D.; Chen, Z.; Hu, X.; Lan, W. Adaptive Dynamic Surface Control for Formations of Autonomous Surface Vehicles With Uncertain Dynamics. IEEE Trans. Control Syst. Technol. 2013, 21, 513-520. [CrossRef]

16. Li, D.; Ma, G.; Li, C.; He, W.; Mei, J.; Ge, S.S. Distributed Attitude Coordinated Control of Multiple Spacecraft With Attitude Constraints. IEEE Trans. Aerosp. Electron. Syst. 2018, 54, 2233-2245. [CrossRef]

17. Feng, Z.; Zheng, W.X. On Extended Dissipativity of Discrete-Time Neural Networks With Time Delay. IEEE Trans. Neural Netw. Learn. Syst. 2015, 26, 3293-3330. [CrossRef]

18. Peng, Z.; Wang, D.; Wang, H.; Wang, W. Coordinated formation pattern control of multiple marine surface vehicles with model uncertainty and time-varying ocean currents. Neural Comput. Appl. 2014, 25, 1771-1783. [CrossRef]

19. Zheng, Z.; Song, S. Autonomous attitude coordinated control for spacecraft formation with input constraint, model uncertainties, and external disturbances. Chin. J. Aeronaut. 2014, 27, 602-612. [CrossRef]

20. Tee, K.P.; Ge, S.S.; Tay, E.H. Barrier Lyapunov Functions for the control of output-constrained nonlinear systems. Automatica 2009, 45, 918-927. [CrossRef]

21. Liu, X.; Ge, S.S.; Goh, C.H. Neural-Network-Based Switching Formation Tracking Control of Multiagents With Uncertainties in Constrained Space. IEEE Trans. Syst. Man Cybern. Syst. 2019, 49, 1006-1015. [CrossRef]

22. Zhang, S.; Tang, Z.; Ge, S.S.; Wei, H. Adaptive neural dynamic surface control of output constrained non-linear systems with unknown control direction. IET Contr. Theory Appl. 2017, 11, 2994-3003. [CrossRef]

23. Wang, W.; Wang, D.; Peng, Z.; Li, T. Prescribed Performance Consensus of Uncertain Nonlinear Strict-Feedback Systems With Unknown Control Directions. IEEE Trans. Syst. Man Cybern. Syst. 2015, 46, 1279-1286. [CrossRef]

24. Park, B.S.; Yoo, S.J. An Error Transformation Approach for Connectivity-Preserving and Collision-Avoiding Formation Tracking of Networked Uncertain Underactuated Surface Vessels. IEEE Trans. Cybern. 2018. [CrossRef] [PubMed]

25. Zhao, Z.; He, W.; Ge, S.S. Adaptive Neural Network Control of a Fully Actuated Marine Surface Vessel With Multiple Output Constraints. IEEE Trans. Control Syst. Technol. 2014, 22, 1536-1543.

26. Bibuli, M.; Singh, Y.; Sharma, S.; Robert, S.; Daniel, H.; Asiya, K. A Two Layered Optimal Approach towards Cooperative Motion Planning of Unmanned Surface Vehicles in a Constrained Maritime Environment. IFAC-PapersOnLine 2018, 51, 378-383. [CrossRef]

27. Singh, Y. Cooperative Swarm Optimisation of Unmanned Surface Vehicles. Ph.D. Thesis, University of Plymouth, Plymouth, UK, 2019.

28. Do, K.D. Synchronization Motion Tracking Control of Multiple Underactuated Ships With Collision Avoidance. IEEE Trans. Ind. Electron. 2016, 63, 2976-2989. [CrossRef] 
29. Sun, H.; Zong, G.; Ahn, C.K. Quantized Decentralized Adaptive Neural Network PI Tracking Control for Uncertain Interconnected Nonlinear Systems with Dynamic Uncertainties. IEEE Trans. Syst. Man Cybern. Syst. 2019. [CrossRef]

30. Sun, J.; Yang, J.; Li, S.; Zheng, W.X. Estimate-Based Dynamic Event-Triggered Output Feedback Control of Networked Nonlinear Uncertain Systems. IEEE Trans. Syst. Man Cybern. Syst. 2019. [CrossRef]

31. Qin, H.; Li, C.; Sun, Y.; Deng, Z.; Liu, Y. Trajectory tracking control of unmanned surface vessels with input saturation and full-state constraints. Int. J. Adv. Robot. Syst. 2018, 15, 1729881418808113. [CrossRef]

32. Li, K.; Tong, S. Observer-based finite-time fuzzy adaptive control for MIMO non-strict feedback nonlinear systems with errors constraint. Neurocomputing 2019, 341, 135-148. [CrossRef]

33. Batmani, Y.; Najafi, S. Event-Triggered Ho Depth Control of Remotely Operated Underwater Vehicles. IEEE Trans. Syst. Man Cybern. Syst. 2019. [CrossRef]

34. Chen, L.; Li, C.; Sun, Y.; Ma, G. Distributed finite-time tracking control for multiple uncertain Euler-Lagrange systems with input saturations and error constraints. IET Control Theory Appl. 2019, 13, 123-133. [CrossRef]

35. Qiu, B.; Wang, G.; Fan, Y.; Mu, D.; Sun, X. Adaptive Sliding Mode Trajectory Tracking Control for Unmanned Surface Vehicle with Modeling Uncertainties and Input Saturation. Appl. Sci. 2019, 9, 1240. [CrossRef]

36. Mu, D.; Wang, G.; Fan, Y.; Sun, X.; Qiu, B. Adaptive LOS Path Following for a Podded Propulsion Unmanned Surface Vehicle with Uncertainty of Model and Actuator Saturation. Appl. Sci. 2017, 7, 1232. [CrossRef]

37. Hu, Q.; Xiao, B.; Shi, P. Tracking control of uncertain Euler-Lagrange systems with finite-time convergence. Int. J. Robust Nonlinear Control 2015, 25, 3299-3315. [CrossRef]

38. Tee, K.P.; Ge, S.S. Control of fully actuated ocean surface vessels using a class of feedforward approximators. IEEE Trans. Control Syst. Technol. 2006, 14, 750-756. [CrossRef]

39. Batmani, Y.; Davoodi, M.; Meskin, N. Nonlinear suboptimal tracking controller design using state-dependent Riccati equation technique. IEEE Trans. Control Syst. Technol. 2016, 25, 1833-1839. [CrossRef]

(C) 2020 by the authors. Licensee MDPI, Basel, Switzerland. This article is an open access article distributed under the terms and conditions of the Creative Commons Attribution (CC BY) license (http:/ / creativecommons.org/licenses/by/4.0/). 